Ann. Zootech., I978, 27, (1), 17-31.

\title{
Etude du besoin en lysine du lapin en croissance en relation avec la concentration énergétique de l'aliment (')
}

\author{
M. COLIN et D. ALLAIN
}

Avec la collaboration technique de G SARDI

Laboratoire de Recherches sur l'Élevage du Lapin, Centre national de Recherches zootechniques, I.N.R.A. 78350 , Jouy-en-Josas (France)

\section{Résumé}

Quatre vingt serze lapins californiens des deux sexes, âgés de 5 semaines en début d'expérience, sont répartis entre 6 régimes correspondant à un schéma factoriel comprenant 3 taux de lysine $(0,45-0,60-0,75$ p. cent) et deux taux de cellulose (ro,o- 7,2 p. cent). Leurs performances de croissance sont étudiées pendant $35 \mathrm{j}$ et le rendement à l'abattage est mesuré à l'issue de l'essai sur la moitié des lapins. En outre, on détermine sur l'autre moitié des animaux ( 8 par régime), le bilan azoté ainsi que l'utilisation digestive et métabolique apparentes des protéines.

L'augmentation du taux de cellulose dans l'aliment (obtenue en remplaçant une partie de l'amidon du régime de base par de la cellulose purifiée) entraîne un abaissement $(-7,5 \mathrm{p}$. cent) de la teneur en énergie digestible des rations mais ne modifie pas l'utilisation digestive apparente de l'azote.

Aux deux concentrations énergétiques, l'apport de lysine supplémentaire améliore la croissance, l'efficacité alimentaire, le bilan azoté, le coefficient de rétention azotée. Mais ces améliorations ne se produisent qu'entre 0,45 et $0,60 \mathrm{p}$. cent de lysine pour les régimes les moins énergétiques alors qu'elles se manifestent également entre $0,60 \mathrm{p}$. cent et $0,75 \mathrm{p}$. cent de lysine dans le cas des aliments les plus énergétiques. Le besoin en lysine du lapin varie donc avec la concentration en énergie digestible de la ration. Pour formuler ce besoin avec une plus grande précision, il convient donc de le rapporter à la teneurr en énergie digestible de l'aliment. Il se situe alors à environ $2,4 \mathrm{~g}$ de lysine pour $\mathrm{I}$ ooo $\mathrm{Kcal}$ d'énergie digestible.

Par ailleurs, l'augmentation du taux de cellulose réduit de $I, 2$ à $I, 6$ point le rendement à l'abattage tandis que l'élévation de la teneur en lysine des aliments semble accroître assez fortement la perte d'ean au ressuage.

\section{Introduction}

Dans un travail précédent (CoLIN, I975a), nous avons montré que lorsqu'ils sont exprimés en pourcentage de la ration, les besoins en acides aminés essentiels du Lapin varient selon la nature du régime de base.

Ainsi, la teneur optimale de lysine fluctue entre $0,55 \mathrm{p}$. cent et $0,70 \mathrm{p}$. cent du

(1) Requests for reprints: Laboratoire de Recherches sur l'Elevage du Iapin, I.N.R.A., B.P. I2, 3I 320 Castanet-lolosan. 
régime. Nous avons alors émis l'hypothèse que, comme pour le Rat (LERNER, I968), le Poulet (Wil,irams et Grau, I956) ou le Porc (RERA'T et al., I970), ce besoin est chez le Lapin d'autant plus élevé que la concentration en énergie digestible de l'aliment est forte. Au cours de la présente étude, nous avons donc cherché à vérifier cette hypothèse et à dégager plus précisément les relations existant entre besoin en lysine du Lapin et teneur en énergie digestible de la ration.

\section{Matẻriel et méthodes}

\section{Présentation de l'expérience}

L'expérience consiste à étudier les performances de 96 Lapins en croissance répartis entre les 6 traitements correspondant à un schéma factoriel comprenant 2 taux de cellulose brute (ro et 17,2 p. cent du régime) et 3 taux de lysine $(0,45-0,60$ et 0,75 p. cent du régime) (tabl. I). La vitesse de croissance et l'efficacité alimentaire sont contrôlées sur l'ensemble des Lapins. En outre, on détermine sur une moitié des animaux (8 par régimes) 1'utilisation digestive apparente des rations et le bilan azoté tandis que sur l'autre moitié, on mesure le rendement à l'abattage.

\section{Animaux}

I es 96 Lapins des 2 sexes de race californienne sont âgés de 35 ou 36 jours en début d'expérience et pèsent alors $869 \pm$ Io $\mathrm{g}$. Les bilans azotés sont établis en plaçant 48 Lapins individuellement dans des cages de collecte (CoI,IN, I974). Les autres animaux sont logés dans des cages individuelles entièrement métalliques munies d'abreuvoirs automatiques à surface d'eau libre. Les répétitions sont constituées d'animaux ayant approximativement le même poids à 4 et à 5 semaines.

\section{Régimes}

Les régimes étudiés (semi-purifiés à base de tourteau de sésame) sont du même type que ceux utilisés a.u Laboratoire dans plusieurs expériences antérieures (COLJN, I974; LEBAS, I975b). La lysine supplémentaire est apportée sous forme de chlorhydrate de lysine et la cellulose additionnelle sous forme de cellulose purifiée fibreuse colmacelle $\mathrm{F}_{2}$ (HENRy et FíTENne, I969).

\section{Conduite de l'expérience}

Les performances de croissance sont étudiées entre 5 et Io semaines selon des modalités déjà décrites (CoI,IN, I975b). La période de collecte permettant la détermination de l'utilisation digestive apparente des rations et celle du bilan azoté (CoLIN, I974) dure II jours et débute 8 jours après le commencement de l'expérience, les animaux restant ensuite dans leur cage jusqu'à l'âge de ro semaines. Les animaux sur lesquels on mesure le rendement à l'abattage sont maintenus dans les conditions expérimentales jusqu'à la date d'abattage. Celui-ci est effectué le même jour pour tous les animaux d'un traitement; il a lieu le premier mercredi 
TABLEAU I

Composition des régimes expérimentaux

Composition of the experimental diets

Taux de cellulose p. cent du régime (Crude fibre p. cent of diet)

Taux de lysine p. cent du régime (Lysine p. cent of diet).

Tourteau de sésame (') (Sesame oil meal) (1) . . . . . . . . . Amidon de maïs (Maize starch) .

Huile d'arachide (Ground mut oil meal)

Paille de blé (wineat straw) .

Cellulose de bois $\left({ }^{2}\right)$ (wood cellulose) $\left(^{2}\right)$. . . . . . . . . .

Chlorhydrate de L-Lysine ( $\left.{ }^{3}\right)$ (I Lysine chlorhydrate) ( $\left.{ }^{3}\right)$

Glutamate de sodium (Sodium ghutamate) . . . . . . . . .

CMV (') (minerals and vitamins) (")

Résultats d'analyse (Analysis).

Matière sèche ( $\mathrm{p}$. cent) (dry matter) $(p . c e n t)$. . . . . . . . . .

Matière organique (p. cent) (organic matter) ( $p . c e n t)$. . . . .

Matière azotée $(\mathrm{N} \times 6,25)$ (p. cent) crude protein) $(N \times 6,25)(p$. cent) .......... . . Cellulose de Wrindel (p. cent) (Crude fiber) (p.cent). . . . . Energie brute (Kcal $/ \mathrm{kg}$ ) Crude energy (Kcal $/ \mathrm{kg}) . . . . . .3844$

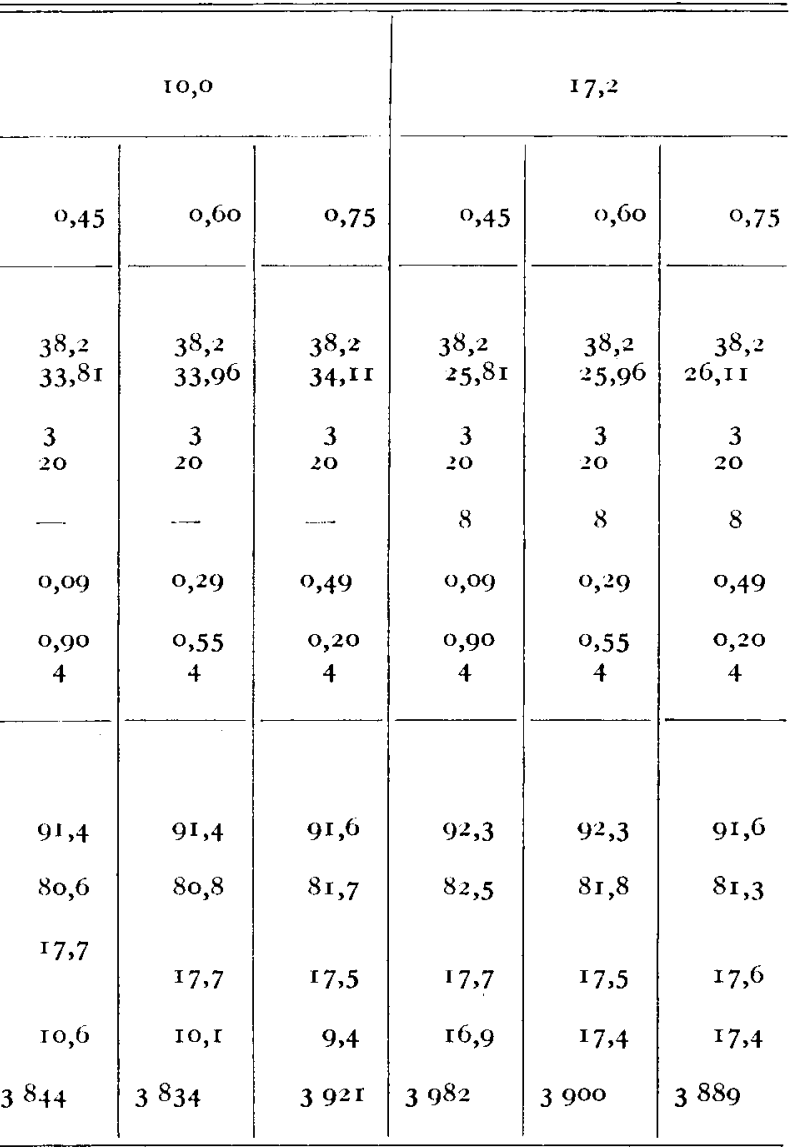

(1) Protéines brutes : 39,7 p. cent. Crude protein : 39.7 p. cent.

(2) Cellulose Colmacel $F_{2}$ (Henky et Étnenne, 1969) contient 94,9 p. cent de matière sèche et $76,4 p$. cent de cellulose de Wrende; commercialisée par Novacrlese (Paris). Cellulose Colmacel $F_{2}$ (Henry and FitnNNE, 1969$)$ containing 94.9 p. cent of dry matter and 76.4 p. cent of crude fibre-commericalised by NovaCELLE (Paris).

(3) Produit commercialisé par Roussizot-Kumlmand (Brevet Ajinomoto) : contient 95 p. cent de

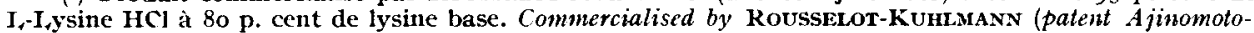
Japan), containing 95 p. cent of l-lysine HCL i.e. 76 p. cent of pure lysine.

(4) Colis, ARkhurst et Lebas (1973).

où le poids moyen des animaux affectés au régime considéré dépasse $2200 \mathrm{~g}$. I,es poids de carcasse correspondent à la présentation française : animal saigné, éviscéré et dépouillé mais avec manchons (LEBAS, 1975a). On calcule :

- le rendement à l'abattage avant ressuage :

$$
\frac{\text { Poids de carcasse chaude après abattage }}{\text { Poids vif }} \times \text { roo }
$$


- le rendement à l'abattage après ressuage :

$$
\frac{\text { Poids de carcasse après ressuage } 24 \mathrm{~h} \grave{\mathrm{a}}+4^{\circ} \mathrm{C}}{\text { Poids vif }} \times 100
$$

\section{Analyse statistique}

L'expérience est réalisée selon un schéma factoriel en blocs complets équilibrés. Pour la plupart des critères, l'exploitation statistique des résultats est assurée par analyse de variance suivie d'un test de NEWManN et KedLs (SNEDECor et CoChRAN, I971). En raison des différences existant sur les poids vifs à l'abattage, les poids de carcasse et les pertes d'eau au ressuage sont étudiés par analyse de covariance (SNedecor et Cochran, I97I) également suivi d'un test de NewMANN et KEUL,S. Enfin, les relations entre poids vif et quantités de lysine ingérées sont étudiées par régression polynomiale (SNEDECOR et COCHRAx, 197I).

\section{Résultats}

\section{I. - Performances de cronssance, de consommation et d'efficacité alimentaire}

Afin de faciliter la présentation des résultats, nous exposerons successivement les effets des variations du taux de lysine sur les performances des lapins, puis les conséquences de l'augmentation de la teneur en cellulose des aliments sur ces performances; enfin, nous envisagerons l'existence d'une éventuelle interaction entre les effets de la lysine et ceux de la cellulose.

Aux 2 taux de cellulose, l'addition de lysine purifiée au régime de base améliore fortement la croissance $(+20 \grave{a}+40 \mathrm{p}$. cent $)$, la consommation $\left(+\mathrm{I}_{5} \mathrm{a}+30\right.$ p. cent) et l'indice de consommation des animaux ( -25 à -7 p. cent) (tabl. 2).

L'effet lysine global est d'ailleurs hautement significatif pour ces 3 critères. L'élévation du taux de cellulose augmente de façon très hautement significative le niveau de consommation $(+14$ à +17 p. cent) et l'indice de consommation $(+5$ à $+\mathrm{I} 5 \mathrm{p}$. cent) des lapins. Il entraîne également un accroissement du gain de poids dans le cas des régimes contenant 0,45 et 0,60 p. cent de lysine $(+$ ro à + I5 p. cent) l'effet cellulose global sur la croissance étant hautement significatif.

. Il convient de remarquer que l'action de la supplémentation en lysine n'est pas la même selon que le régime contient Io,o ou I 7,2 p. cent de cellulose. Ainsi, la croissance maximale est obtenue avec $0,75 \mathrm{p}$. cent de lysine dans le cas du régime à $10,0 \mathrm{p}$. cent de cellulose, et avec seulement $0,60 \mathrm{p}$. cent de lysine lorsque la ration apporte I7,2 p. cent de cellulose. De plus, les conséquences de la carence en lysine sont moins accentuées avec 17,2 p. cent de cellulose qu'avec Io p. cent par rapport aux résultats obtenus avec 0,75 p. cent de lysine; l'absence de toute supplémentation en -lysine entraîne des réductions de la vitesse de croissance de $19 \mathrm{p}$. cent et 29 p. cent" respectivement pour I 7,2 et ro p. cent de cellulose.

Il existe donc une interaction entre l'action des variations du taux de lysine et celle de l'élévation de la teneur en cellulose : cette interaction est d'ailleurs significative au seuil $\mathrm{P}=0$, Io. Des phénomènes comparables sont observés pour l'indice de consommation. $\mathrm{L}$ interaction est cette fois significative au setuil $\mathrm{P}=0,05$. Par contre, on n'observe pas d'interaction pour la consommation. 
L'élévation du taux de cellulose entraîne une augmentation de l'appétit des lapins; ceux-ci ingèrent donc à taux de lysine égal, cet acide aminé en quantités d'autant plus fortes que la ration contient plus de cellulose. On peut alors chercher une relation entre la croissance des lapins et la dose de lysine ingérée.

Une étude réalisée sur l'ensemble des 96 résultats individuels montre qu'une équation de régression du $2^{e}$ degré rend très bien compte de l'évolution du gain de poids des lapins en fonction de la lysine ingérée (fig. I).

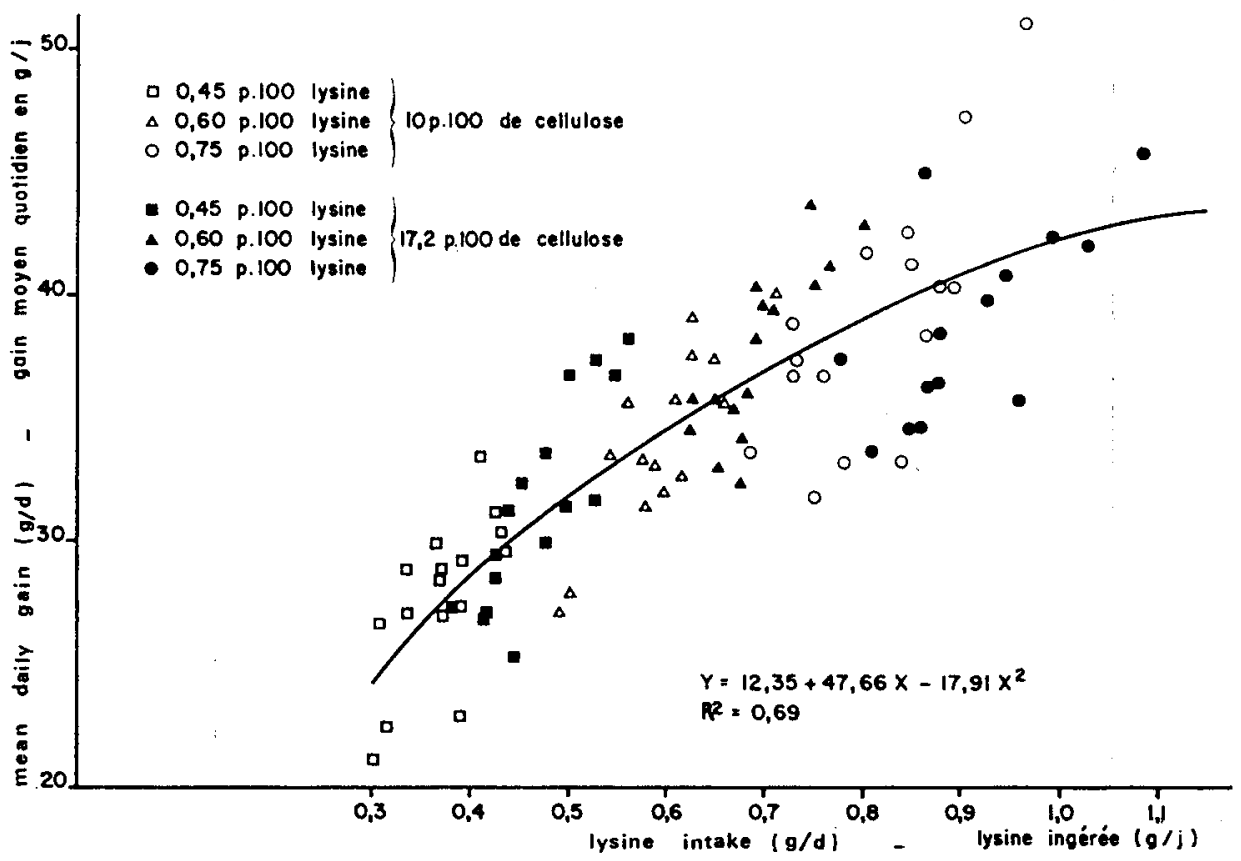

FIG. I. - Relation entve le gain de poids moyen quotidien et la quantité de lysine ingérée (végression établie d'après les résultats individuels de la présente étude).

Relation between the daily weight gain and daily intake of lysine (regvession established according to the individual results of this experiment).

$\mathrm{Y}=\mathrm{I} 2,35+47,66 \mathrm{X}-\mathrm{I} 7,9 \mathrm{IX} 2(\mathrm{R} 2=0,69)$.

$\mathrm{F}$ linéaire $197,2 * * *$.

F. Quadratique $4,05 *$.

$\mathrm{Y}$ Gain de poids quotidien $(\mathrm{g} / \mathrm{j})$.

$\mathrm{X}$ Quantité de lysine ingérée $(\mathrm{g} / \mathrm{j})$.

Un ajustement par un polynôme du $3^{\mathrm{e}}$ degré ne diminue pratiquement pas la somme des écarts à la régression et s'avère donc inutile.

\section{2. - Utilisation digestive apparente et teneur en énergie digestible des aliments}

L'apport de cellulose purifiée dans les rations expérimentales déprime ( $P$ $<0,00$ I) la valeur des coefficients d'utilisation digestive apparente de la matière sèche, de la matière organique et de l'énergie de 7 à 8 points (tabl. 3). Par contre, 


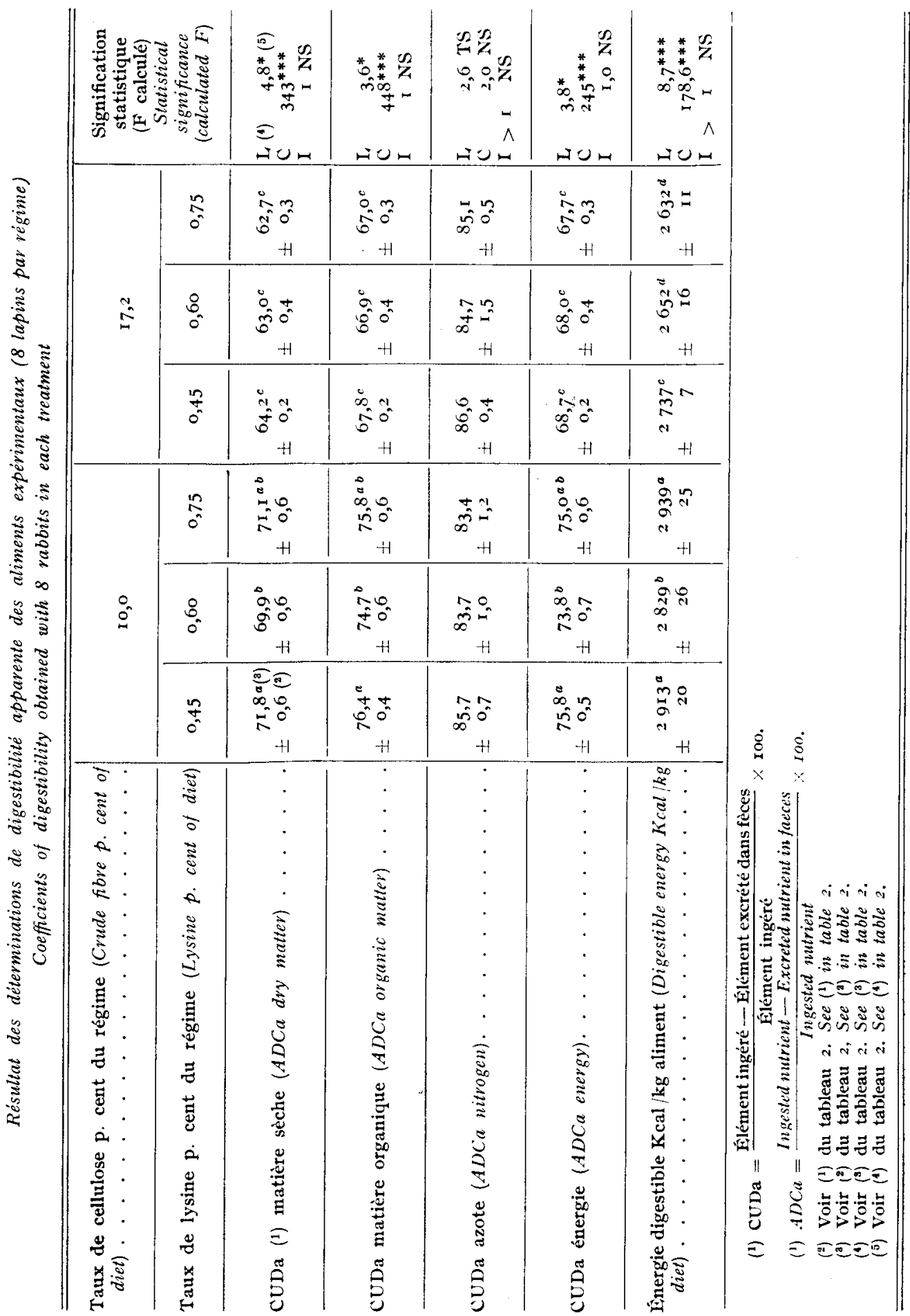


les variations du taux de lysine n'entraînent pour ces mêmes critères que des diffé rences d'intensité très faibles bien que significatives dans le cas des aliments contenant io p. cent de cellulose. Toutefois, dans ce dernier cas, les écarts observés sur les CUDa ne suivent pas les variations provoquées du taux de lysine. Enfin, les coefficients d'utilisation digestive apparente de l'azote ne varient que très faiblement avec le taux de lysine ou de cellulose des rations.

Les résultats obtenus permettent de déterminer directement l'énergie digestible des aliments expérimentaux. Comme prévu, le remplacement de 8 points d'amidon par de la cellulose de bois entraîne un abaissement de la teneur en énergie digestible des aliments (environ - $220 \mathrm{Kcal} \mathrm{ED} / \mathrm{kg}$ d'aliment). Les fluctuations avec le taux de lysine sont beaucoup plus faibles. Par ailleurs, l'étude des quantités d'énergie digestible ingérées par les lapins montre que, dans le cas des rations contenant 0,75 p. cent de lysine, les animaux consomment une quantité d'énergie digestible identique que l'aliment apporte 2632 ou $2939 \mathrm{Kcal} / \mathrm{kg}$ (tabl. 3 ).

Par contre, avec 0,45 et $0,60 \mathrm{p}$. cent de lysine, la consommation d'énergie digestible par les Lapins est la plus forte pour le régime le plus dilué en énergie digestible.

La différence est d'autant plus accentuée que l'aliment est pauvre en lysine $(+\mathrm{I} 8 \mathrm{p}$. cent avec 0,45 p. cent de $1 \mathrm{ysine}$; + Io p. cent avec 0,60 p. cent $)$. L'interaction entre les effets de la teneur en lysine et ceux de la concentration énergétique sur l'ingestion d'énergie digestible par les lapins est d'ailleurs très hautement significative (tabl. 3).

\section{3. - Bilan azoté et utilisation métabolique des matières azotées}

Le bilan azoté évolue de façon comparable au gain de poids. Il est amélioré par une supplémentation des régimes par la L lysine purifiée et cet effet est d'autant plus accentué que la concentration énergétique de la ration est élevée (tab1. 4). De même, l'apport de lysine entraîne une augmentation du coefficient de rétenticn azotée et du coefficient d'utilisation pratique de l'azote. A taux de lysine égal, on n'observe pratiquement aucune différence due à la variation du taux de cellulose.

\section{4. - Poids de carcasse et rendements à l'abattage}

Selon les traitements expérimentaux, les lapins sont abattus à Io semaines d'âge (les 2 régimes à $0,75 \mathrm{p}$. cent de lysine), i I semaines (les 2 régimes à $0,60 \mathrm{p}$. cent de lysine et le régime à $0,45 \mathrm{p}$. cent de lysine et $17,2 \mathrm{p}$. cent de cellulose), I2 semaines (régime à $0,45 \mathrm{p}$. cent de lysine et I0,0 p. cent de cellulose) (tabl. 5). Les poids vifs des animaux à l'abattage sont en moyenne pratiquement identiques pour les 3 aliments contenant Io,o p. cent de cellulose et pour celui à $I_{7,2} \mathrm{p}$. cent de cellulose et 0,75 p. cent de 1ysine; ils sont par contre, plus faibles pour les animaux ayant reçu la ration à $\mathrm{I} 7,2$ p. cent de cellulose et $0,45 \mathrm{p}$. cent de lysine; plus forts pour les lapins recevant le régime à $I 7,2$ p. cent de cellulose et 0,60 p. cent de lysine.

Afin d'atténuer d'éventuels effets de ces différences sur le poids vif, les poids de carcasse avant et après ressuage, de même que la perte d'eau au ressuage sont étudiés par analyse de covariance. Après ajustement pour un poids vif de $2308 \mathrm{~g}$, le poids de carcasse avant ressuage est significativement augmenté ( + I7 g) quand le taux de lysine de la ration croît, il est abaissé (différence significative au 


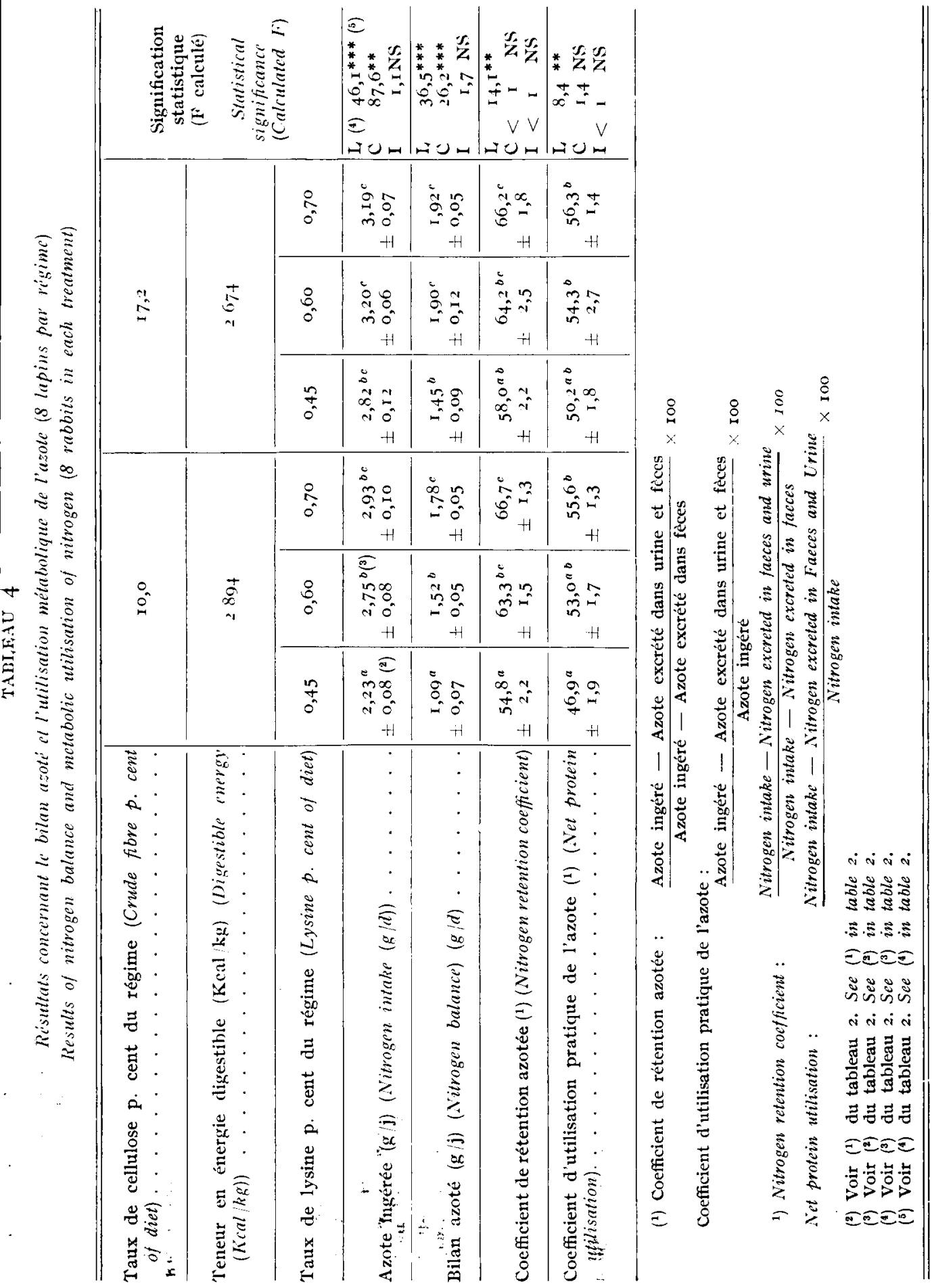


M. COLIN, D. ALLAIN

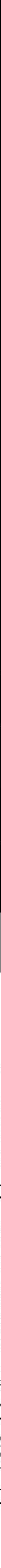


seuil $\mathrm{P}<\mathrm{o}, \mathrm{I}$ ) de $\mathrm{I} 8$ à $33 \mathrm{~g}$ lorsqu'on incorpore à l'aliment la cellulose purifiée. Globalement, le rendement à l'abattage avant ressuage est donc d'autant meilleur que l'apport de lysine dans la ration est plus élevé et que sa teneur en cellulose est plus faible.

La perte d'eau lors du ressuage augmente très fortement $(+30$ à + roo $\mathrm{p}$. cent) lorsque le taux de lysine dans l'aliment croît $(\mathbf{P}<0,00 \mathrm{I})$. Le poids de carcasse après ressuage ajusté pour un poids vif de $2308 \mathrm{~g}$ varie également significativement avec le taux de lysine. Mais l'effet est différent selon que l'aliment est riche ou pauvre en cellulose (interaction hautement significative). Les carcasses ressuées les plus lourdes sont obtenues pour $0,45 \mathrm{p}$. cent de lysine lorsque le régime contient ro,o p. cent de cellulose et pour o,6o p. cent de lysine lorsqu'il en apporte I 7,2 p. cent. L'effet cellulose global sur le poids des carcasses après ressuage reste très faible. Ces différences se retrouvent au niveau des rendements à l'abattage après ressuage.

\section{Discussion}

\section{I. -- Variation du taux de cellulose et teneur en énergie digestible des régimes}

L'élévation du taux de cellulose entraîne une très nette chute de l'utilisation digestive apparente de la matière sèche, de la matière organique et de l'énergie mais ne provoque aucune modification de l'utilisation digestive apparente de l'azote. Ces résultats sont en parfait accord avec ceux de LEBAS (1975 $b$ ) obtenus avec des régimes de même type.

L'addition de 7,2 p. cent de cellulose à l'aliment entraîne une baisse de la digestibilité de la matière organique de 8,3 points et de la teneur en énergie digestible de l'aliment de $259 \mathrm{Kcal} / \mathrm{kg}$ de matière sèche. Or la cellulose additionnelle étant digestible par le Lapin à I 5 p. cent environ (CoL,IN et ALLAIN, données non publiées), le remplacement de l'amidon par la cellulose purifiée représente une augmentation du taux de glucides indigestibles de l'aliment de 6, I points environ. Cette variation entraîne une diminution théorique de 7,4 points de la digestibilité de la matière organique et de $250 \mathrm{Kcal} / \mathrm{kg}$ de matière sèche de la teneur en énergie digestible de l'aliment, valeurs très proches des différences réellement mesurées. Il semble donc que l'apport de cellulose purifiée dans les régimes provoque principalement un effet de dilution sans affecter la digestibilité apparente des autres éléments de la ration. Cette hypothèse, déjà formulée par LEBAS (I975a) semble confirmée par le fait que l'augmentation du taux de cellulose ne modifie pas la digestibilité apparente des protéines, phénomène déjà signalé par HoovE, et HeITMANN (I972), LEBAs (I975b) et dans certaines limites par ABGAROWicz (I948) et ERIKSSON (I952).

En conclusion, dans nos conditions expérimentales, l'augmentation du taux de cellulose peut être considérée comme un apport de lest inerte dont les effets semblent limités à un abaissement de la teneur en énergie digestible des aliments.

\section{2. - Iariations du taux de lysine}

Les résultats obtenus confirment le fait déjà signalé (CoLIN, I973 et I974) qu'un régime semi-purifié à base de tourteau de sésame est carencé en lysine. Fin outre, comme dans ces travaux antérieurs, la supplémentation en lysine aug- 
mente la croissance, la consommation, 1'efficacité alimentaire, le bilan azoté et l'utilisation métabolique de l'azote.

Dans nos conditions expérimentales, le besoin en lysine du Lapin en croissance exprimé en pourcentage du régime se situe à $0,60 \mathrm{p}$. cent environ lorsque l'aliment apporte 2600 à $2700 \mathrm{Kcal} / \mathrm{kg}$ d'énergie digestible, mais paraît très nettement supérieur à ce taux lorsque l'énergie digestible de la ration est de $2800-2900 \mathrm{Kcal} / \mathrm{kg}$. Il existe done bien, comme nous en avions émis 1'hypothèse (CoLIN, I975a), une relation entre le besoin en lysine du lapin, exprimé en pourcentage de l'aliment, et la densité énergétique de celui-ci. Il semble done plus précis de formuler le besoin en lysine du Lapin en le rapportant à l'énergie

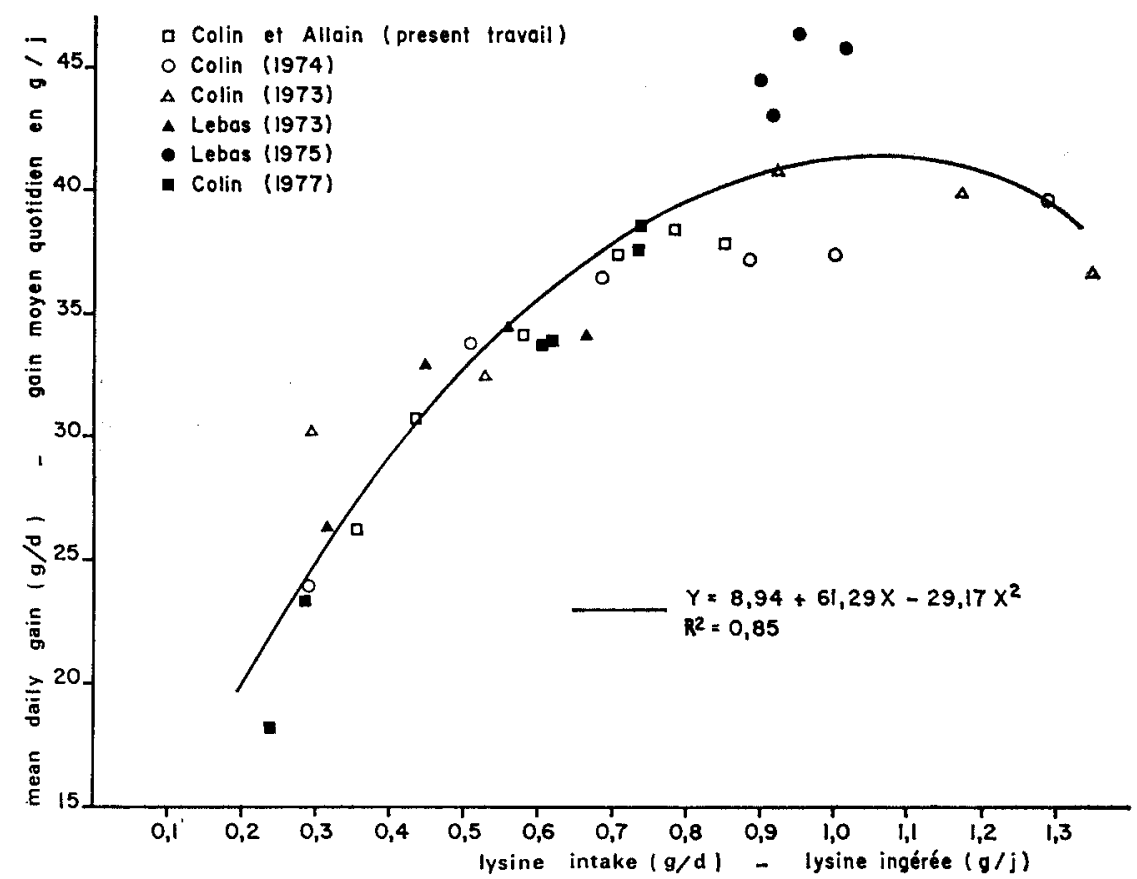

FIG. 2. - Relation entre le gain de poids moyen quotidien et la quantité de lysine ingérée (végvession établie d'après l'ensemble des résultats moyens des expériences réalisées au laboratoire). Relation between the daily weight gain and the daily intake of lysine (regression established according to all experimental means obtained in the laboratory).

digestible de l'aliment, d'après l'expérience présentée ici, ce besoin est compris entre 2,3 et $2,5 \mathrm{~g}$ de lysine pour I ooo Kcal d'énergie digestible.

En fait, les relations entre besoin en lysine du Lapin et densité énergétique de la ration paraissent dues au fait que, dans certaines limites (ABGARowICz, I948; LEBAS, I975b), le Lapin consomme d'autant plus de granulé que celui-ci apporte peu d'énergie digestible. Dans le présent travail, on observe d'ailleurs que, lorsque les régimes sont équilibrés en lysine, les lapins ajustent rigoureusement l'ingestion d'aliment sur la concentration en énergie digestible de celui-ci, phénomène déjà signalé chez le Lapin (ERIKSSON, I952; LEBAS, I975b), le Chien (CoGwILL, I928), le Rat (Henry et Rerat, I966), le Porc (Rera't et al., 1970). Compte tenu de ces observations, pour un taux donné de lysine dans l'aliment, le Lapin ingère donc cet 
acide aminé en quantités d'autant plus importantes que la concentration en énergie digestible du régime est plus faible. La distribution d'une ration pauvre en lysine n'aura donc pas les mêmes effets sur la croissance selon que cette ration sera plus. ou moins énergétique. Nous retrouvons chez le Lapin (fig. I) les relations entre croissance (ou bilan azoté) et quantités d'acides aminés indispensables ingérées, relations mises en évidence chez le Porc par RERAT et Lougnon (I966). L'étude des performances obtenues dans notre laboratoire avec des régimes semi-purifiés à base de tourteau de sésame (COLIN, I973, I974 et I977; présente étude; LEBAS, I 973 et $1975^{b}$ ) permet d'illustrer ces liaisons. Pour ce travail ont été exclus quelques régimes dans lesquels un facteur limitant autre que la lysine a été identifié (taux protéique LEBAS, I973 et COLIN, I974; concentration énergétique LEBAS, I975; teneur en chlore CoLIN, I977). Dans ces conditions, le gain de poids moyen quotidien des lapins est en très grande partie expliqué par la quantité de lysine ingé rée (fig. 2) selon l'équation de regression :

$\mathrm{Y}=8,9+6 \mathrm{I}, 2 \mathrm{X}-29,2 \mathrm{X}^{2}\left(\mathrm{R}^{2}=0,85\right)$.

$\mathrm{F}$ linéaire $129^{* * *}$.

F quadratique $40^{* * *}$.

$Y$ Gain moyen quotidien ( $\mathrm{g} / \mathrm{j})$.

$X$ Quantité de lysine ingérée $(g / j)$.

D'après ces résultats, le besoin en lysine du Lapin en croissance se situe à $\mathrm{I} g / \mathrm{j}$ environ. Cependant, des croissances de haut niveau sont déjà obtenues pour une consommation quotidienne de $0,75 \mathrm{~g}$ de lysine. Au-dessus de cette quantité, l'efficacité d'utilisation marginale de la lysine apportée devient très faible ( Io g de gain de poids par gramme de lysine ingérée). D'un point de vue pratique, il convient. donc de distribuer des aliments assurant une ingestion moyenne de lysine de $0,75 \mathrm{~g} / \mathrm{j}$. Selon que l'aliment est concentré en énergie digestible (2 g00 Kcal $/ \mathrm{kg})$ ou au contraire plus dilué ( $265^{\circ} \mathrm{Kcal}$ ), ce niveau de consommation est obtenu pour un taux de lysine dans la ration de 0,60 ou de 0,70 p. cent, soit environ $2,4 \mathrm{~g}$ ! I ooo $\mathrm{Kcal} / \mathrm{kg}$ d'énergie digestible.

\section{3. - Rendements à l'abattage et pertes au ressuage}

L'augmentation du taux de cellulose entraîne une légère baisse du rendement à l'abattage avant et après ressuage (respectivement $\mathrm{I}, 6$ et $\mathrm{I}, 2$ points). Ce phénomène déjà observé par LEBAS (I975a) peut s'expliquer par un développement plus important du tube digestif dans le cas d'un régime riche en cellulose, conformément à ce qu'a observé HeNRY (I969) chez le Porc. Il faut cependant souligner que malgré l'importante différence entre les taux de cellulose des aliments, la dégradation des rendements à l'abattage reste faible.

Par ailleurs, on observe, d'un traitement à l'autre, des variations très importantes de la valeur de la perte en eau au ressuage. Ce résultat doit être interprété avec la plus grande prudence.

En effet, les animaux étant abattus à des dates différentes selon le régime qu'ils reçoivent, les écarts enregistrés peuvent refléter au moins partiellement, des modifications dans les conditions de ressuage. Il conviendra donc d'entreprendre des études plus systématiques de ce phénomène, en recherchant notamment si l'abattage de lapins plus jeunes, par suite d'une amélioration de la vitesse de croissance par voie nutritionnelle n'entraîne pas une dégradation de pouvoir de rétention en eau de la viande (OuHAyoun et al., r973). 


\section{Conclusion}

En conclusion, ce travail montre que les besoins en acides aminés essentiels du Irapin, comme ceux du Porc (RERAT et al., I970) doivent, pour être formulés avec précision, être rapportés à l'énergie digestible du régime. Dans nos conditions expérimentales, le besoin en lysine se situe à environ $2,4 \mathrm{~g} / \mathrm{x}$ ooo Kcal d'énergie digestible. Des études similaires devront être entreprises pour les acides aminés soufrés dont l'importance dans l'alimentation de cet animal a souvent été signalée (Coirin, Arkhurst et Lebas, i973; Adamson et Fisher, I973; Colin, I975; Davidsox et Spreadbury, I975).

fccepti pour publication en novembre 1977.

\section{Summary \\ Lysine requirement of the growing rabbit as related to the energy concentration of the diet.}

A total of 96 Californian rabbits of both sexes and aged 5 weeks at the beginning of the experiment, were allocated to 6 treatments corresponding to a factorial design including 3 lysine levels $(0.45-0.60-0.75$ p. cent) and two crude fibre levels (ro.o - I 7.2 p. cent). Their growth per formance was studied during 35 days and the carcass yield measured in half of the animals at slaughter. Nitrogen retention as well as the apparent digestibility and metabolic utilization were determined in the other half of the animals ( 8 per treatment).

Increase in the crude fibre content of the diet (obtained by replacing part of the dietary starch by purified crude fibre) led to a lowering ( $-7.5 \mathrm{p}$. cent) of the digestible energy content of the diets, but did not change the apparent digestibility of protein.

At the two energy concentrations, the addition of supplementary lysine improved growth, feed efficiency, nitrogen balance and nitrogen retention coefficient. However, for the diets with the lowest energy content, these improvements only occurred between lysine levels ranging from 0.45 to $0.60 \mathrm{p}$. cent, whereas they were also observed at lysine levels ranging from 0.60 to $0.75 \mathrm{p}$. cent in the case of the diets with the highest energy content. Accordingly the lysine requirement of the rabbit varies with the digestible energy concentration of the diet. A more accurate definition of this requirement should therefore be made in relation to the digestible energy level of the diet. It then represents around $2.4 \mathrm{~g}$ lysine per $\mathrm{I}$ ooo kcal digestible energy.

Increase in the crude fibre level reduced the carcass yield by 1.2, I.6 points, while a rise in the dietary lysine level seemed to strongly enhance the loss of water during shrinkage.

\section{Références bibliographiques}

ABgakowicz F., 1948. Untersuchungen über den Linfluss des Ballastes in der Nahrung des Kaninchens. Thèse Univer. Zürich, I $948,7^{8}$ pages.

ADAMSON I., FISHER H., I973. The amino acid requirement of the growing rabbit: an estimate of quantitative needs. J. Nutr., 103, r $306-$ I 3 ro.

CoLrs M., 1973. Influence de l'addition de lysine à des régimes à base de tourteau de sésame chez le lapin. J. Rech. avicoles et cunicoles, I9-2I-I.T.A.V.I. éd. Paris.

Corrs M, r974. Supplémentation en lysine d'un régime à base de tourteau de sésame chez le lapin. Eiffets sur les performances de croissance et le bilan azoté estimé par deux méthodes. Ann. Zootech., 23, I I9-132.

COLIx M., 1975a. Effets sur la croissance du lapin de la supplémentation en L-lysine et en DLméthionine de régimes végétaux simplifiés. Ann. Zootech., 24, 465-474. 
CoLn M., r.975b. Eiffet de la teneur en arginine du régime sur la croissance et le bilan azoté chez le lapin : relation avec le taux de lysine. Ann. Zootech., 24, 629-638.

CoLIN M., I977. Effet d'une variation du taux de chlore dans l'alimentation du lapin en croissance. Ann. Zootech. 26, 99-103.

COLIN M., Arkiurst G., LEBAS F., I973. Effets de l'addition de méthionine au régime alimentaire sur les performances de croissance chez le lapin. Ann. Zootech., 22, 485-49r.

Cowgil. G. R., I928. The energy factor in relation to food intake: experiments on the dog. Am. J. Physiol., 85, 45-64.

Davidson J, Spreadiury D., 1975. Nutrition of the new-zealand white rabbit. Proc. Nutr. Soc., 34, $75-83$.

ERIKSSON S., I952. Metabolism of rabbits at different levels of crude fiber and protein Almquist and Wiksells Boktryckers A B, Uppsala (Suède).

HenRy Y., ÉtienNe: M., ig69. Effets nutritionnels de l'incorporation de cellulose purifiée dans le régime du porc en croissance-finition. I. Influence sur l'utilisation digestive des nutriments. Ann. Zootech., 18, 337-357.

HENRy Y., r969. Effets nutritionnels de l'incorporation de cellulose purifiée dans le tégime du porc en croissance-finition. II. Influence sur les performances de croissance et la composition corporelle. Ann. Zootech., 18, 371-384.

HENRY Y., RFRAT A., 1966. Évolution de l'ingestion spontanée de principes énergétiques en fonction de la vitesse de croissance et de la protéinogénèse chez le rat blanc, in Cahier $A$.E.C. no 6 : "Aminoacides, Peptides, Protéines ", $24 \mathrm{I}-262$.

Hoover W. H., Heitmans R. N., 1972. Effects of dietary fiber levels on weight gain cecal volume and volatile fatty acid production in rabbits. J. Nutr., 102, 375-380.

LEBAS F., 1973. Effet de la teneur en protéines de la ration à base de soja ou de sésame sur les performances de croissance chez le lapin. Ann. Zootech., 22, 83-92.

I.EBAS F., I975a. Le lapin de chair, ses besoins nutritionnels et son alimentation pratique. Édition revue et complétée. Mars $1975,5^{\circ}$ p., I.T.A.V.I., éd. Paris.

LEBAS $\mathbf{F}$, I975 b. Influence de la teneur en énergie de l'aliment sur les performances de croissance chez le lapin. Ann. Zootech., 24, $28 \mathrm{I}-288$.

LERNER J. T., r968. Contribution à l'étude du besoin en lysine en relation avec le taux énergétique chez le Rat et le Porc. Thèse Doc. $3^{\mathrm{e}}$ cycle, Paris, Fac. Science.

Ouhayoun J., Rouvizir R., Valin C., LacourT A., I973. Variations génétiques de l'évolution post-movtem du pH du tissu musculaire du Lapin. J. Rech. avicoles et cunicoles, 75-78. I.T.A.V.I. éd. Paris.

RERAT A., LERNER J., HENRY Y., Bourdon D., I97o. Étude du besoin de lysine du potc en croissance en relation avec le taux énergétique du régime. Journées Rech. Porcine en France, I.N.R.A., Paris, 79-84.

Rerat A., LoUgNoN J., rg66. Les besoins en amino-acides du porc en croissance (revue) in Cahier A.E.C. no 6, "Amino-acides, peptides, protéines ", 344-422.

SNEDFCOR G. W., COCHRAN W. G., I97I. Méthodes statistiques, Association de coordination technique agricole, Paris (649 p.).

Williams M. A., GraU C. R., I956. Food intake and utilization of lysine deficient protein by the chick in relation to the digestible energy concentration of the diet. $J . N u t r ., 59,243-265$. 